\title{
Changes in microstructure of snow under large deformations
}

\author{
Michael Q. Edens and Robert L. BROWN \\ Department of Civil and Agricultural Engineering, Montana State University, Bozeman, \\ Montana 59717-0007, U.S.A.
}

\begin{abstract}
A set of microstructural variables is selected to characterize the behavior of snow. Corresponding mathematical relations from quantitative stereology theory are presented along with relations and techniques required for numerical evaluation. An experimental investigation is carried out to determine changes in these variables for snow subjected to large compressive deformations. The microstructural variables studied included coordination number, grain-size, bond radius, neck length, pore-size, free surface area and grains/unit volume. Measurements at several stages of deformation are used to evaluate the changes in the microstructure as functions of deformation. Microstructure measurements of six snow samples subjected to confined compression tests are presented for pre-compressed and compressed states, corresponding to final stresses of $0.387,0.77$ and $1.55 \mathrm{MPa}$. Grain-size and bond radius were found to go through finite changes during compression, although the variation of bond radius was more complicated in nature. The coordination number and number of bonds/unit volume were found to go through large changes during compression, while specific free surface area was found to increase by $100 \%$ due to grain- and bond-fracture processes. No discernible patterns of change in neck length could be found in the experiments. A close relationship between some of the microstructural variables and the stress response of the material was observed. These results serve to contribute to the presently available data and understanding of the microstructural behavior of snow.
\end{abstract}

\section{INTRODUCTION}

The mechanical properties of snow can exhibit a strong influence on many winter activities such as vehicle travel, winter recreation, and avalanche prediction and control. One of the most commonly observed features of snow is its compressibility. It can undergo large volumetric deformations when subjected to moderate bulk stresses. These deformations are basically irreversible. Its ability to undergo large deformation is in large part due to its porosity. In its natural state, snow exhibits a wide range of grain shapes and densities. Abele and Gow (1975, 1976) have demonstrated the effect initial snow density has on its load-bearing ability. From their experiments, it was clearly demonstrated, that for undisturbed snow with densities from 100 to $300 \mathrm{~kg} \mathrm{~m}^{-3}$ and subjected to high-rate deformation, lower initial densities resulted in lower final densities for a given final stress than that with a higher initial density. They attributed this to the irregular-shaped grains found in low-density snow and the more uniform rounded nature of the higher-density material. It was found that the initial density was a significant factor for final stresses $<1 \mathrm{MPa}$. Beyond this, the effects of higher initial density became less obvious.
Their studies also showed the rate-dependent nature of the load-bearing ability of snow. These results have also been verified with a constitutive theory (Brown, 1979, 1980).

Modern constitutive theories are now using microstructural parameters, which may include bond radius, neck length, grain-size and three-dimensional coordination number, among others. The bond is the minimum constriction in the necked region connecting two grains. The neck is defined as the total constricted region between the two grains. It is assumed to begin at the point where the surface curvature changes from convex outward to concave outward as viewed in a surface section. In cases where a sharp change in surface slope exists, this is used to define the juncture of the neck and grain. Figure 1 illustrates a neck connecting two grains, and Figure 2 illustrates a surface section with a number of grains. Admittedly, the definition used here to define the neck contains a degree of subjectivity and at times is difficult to implement. The three-dimensional coordination number is simply the average number of bonds per grain in the material.

A volumetric constitutive law based on a neck-growth model was developed by Brown (1980). This model also 


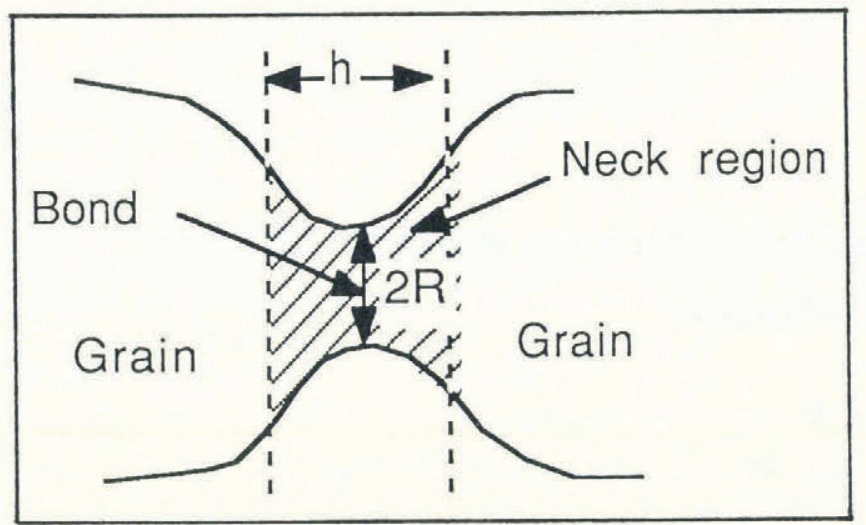

Fig. 1. Schematic of neck and bond as they appear in a surface section.

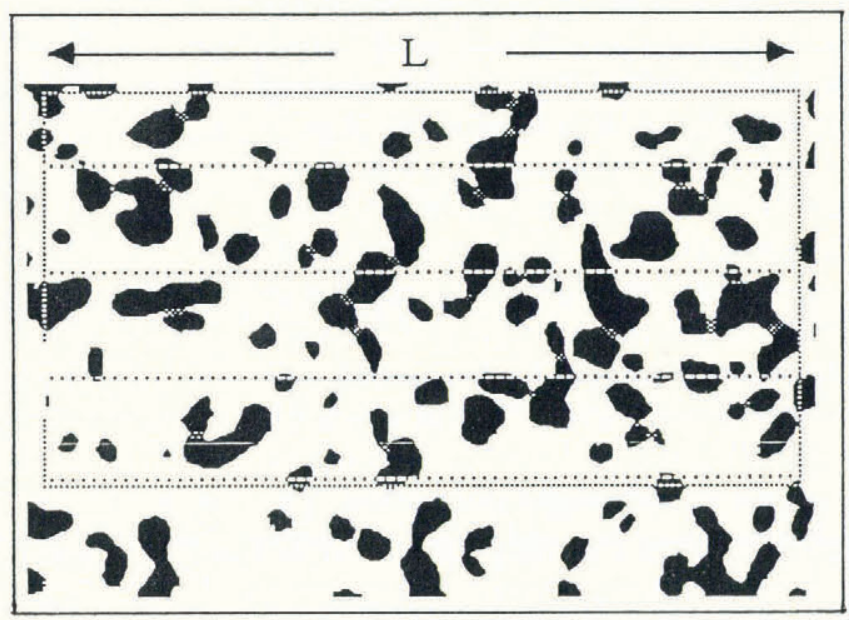

Fig. 2. Example of basic counting techniques. Twenty-one grains are intercepted for a total line length of $4 \times L$.

included structural parameters for change in neck radius, neck length, grain radius and coordination number. The resulting equation described qualitatively the behavior for snow subjected to arbitrary deformation histories for a range of initial densities. In order to obtain accurate quantitative description of the strains, empirical adjustment was required. A more general constitutive law based upon non-equilibrium thermodynamics and the mechanical properties of ice has been developed by Hansen and Brown (1986) for snow subjected to highrate multi-axial deformations. The microstructural properties of snow in this theory are included in the form of internal-state variables. This theory was shown to predict behavior such as rate-dependence, stress relaxation and strain recovery. Over the past 10 years, other investigators have formulated constitutive relations with the use of microstructural variables. This includes Maeno and Ebinuma (1983), Ebinuma and Maeno (1985), Alley (1987), Wilkinson (1988) and others.

Accuracy of any constitutive relation is dependent upon understanding the behavior of the microstructural variables included in the theory. Data are needed for the evaluation of empirical parameters. In addition, most constitutve laws based on microstructural parameters are too complicated to be of use in practical applications. Simplifications of these constitutive laws for special sit- uations are best accomplished with knowledge of which microstructural parameters can be eliminated. At the present time, little or no data on microstructural behavior exist for snow densified by large deformation to densities in the $500-650 \mathrm{~kg} \mathrm{~m}^{-3}$ range. The work of Abele and Gow $(1975,1976)$ provides a strong basis for understanding bulk behavior of snow subjected to high deformation rates and large strains, but little or no data were obtained relating the microstructure to the deformation. Gubler (1978a, b) and Kry (1975a, b) carried out extensive microstructural evaluation in their work, but these studies were generally confined to final densities below $500 \mathrm{~kg} \mathrm{~m}^{-1}$ and to small deformations.

It is the major purpose of this investigation to evaluate the changes in microstructural variables that result from large deformations. In addition, it is intended to measure microstructural variables which until the present have not been adequately measured and mathematically described. An empirical equation relating the number of bonds per volume to the mean pore length will be given and its physical significance discussed. A new definition of band width in Gubler's (1978b) work will also be given. The results obtained will contribute to currently available data and to the understanding of the microstructural processes responsible for material deformation in granular materials.

\section{STEREOLOGY AND MICROSTRUCTURAL PARAMETERS}

Stereology is a statistically based method by which quantities measured directly from a two-dimensional plane intersecting a three-dimensional body are used to obtain average values for the three-dimensional structure of the body. The relationships between two and three dimensions, and the resulting values, can then be used as a means for understanding the average microstructural properties of the body. The task then is to select those parameters most important to characterizing the material behavior and making the pertinent section measurements. The variables selected to represent the microstructure of snow under large deformations must be able to characterize the average re-arrangement of the microstructure. These variables must be able to represent dominant mechanisms such as pore collapse, bond fracture, intergranular glide, and neck and bond growth.

Measurements from a section plane are generally based upon three counting methods: point, lineal and areal. The main assumptions necessary for validity of measurenents are homogeneity and isotropy. Hansen (1985) considered the microstructure of snow by representing it as a three-phase mixture. The phases were the air, neck and ice-grain phases. The subscripts used to represent each phase are respectively $\mathrm{a}, \mathrm{b}$ and $\mathrm{g}$. The required measurements of each phase in the section plane are represented by the following variables:

$N_{\text {gL }}$ Number of grains intercepted per unit length of test line.

$N_{\text {bA }}$ Number of bonds per unit area.

$N_{\mathrm{gA}}$ Number of grains per unit area.

$A_{\mathrm{aA}}$ Area fraction of air phase appearing in the surface section. 
$A_{\mathrm{bA}}$ Area fraction of neck phase in the surface section. $A_{\mathrm{gA}}$ Area fraction of ice grain phase in the surface section.

The remaining surface-section measurements required are:

$\alpha$ Ratio of ice density to snow density, $\rho_{\mathrm{i}} / \rho_{\mathrm{s}}$.

$D_{2}$ Mean bond diameter.

$E$ Harmonic mean of bond diameter.

$f 0$ Fraction of grains with 0 bonds appearing in the section plane.

$f 1$ Fraction of grains with 1 bond appearing in the section plane.

$f 2$ Fraction of grains with 2 bonds appearing in the section plane.

$f 3$ Fraction of grains with 3 bonds appearing in the section plane.

The definition used for a grain bond is that given by Kry (1975a). A grain bond intercepted by the section plane will appear as a line joining two opposite edges of the ice in the plane. To identify this line as a bond, three criteria must be met. First, there must be a minimum constriction of $67 \%$ of the ice in the section plane. Secondly, the constriction must be present on both edges of the ice. Finally, the constrictions must point approximately towards each other. In practice, applying this definition will involve some subjectivity. As long as the consistency in applying the definition is observed, comparison of measurements should not be seriously affected.

The neck is considered to be that part of the ice joining two ice grains and lying between the points where the surface goes from convex to concave with respect to the outward normal. The application of this definition also contains an element of subjectivity, since identifying the point of change in curvature sign is difficult. The neck regions tend to contain less than $2 \%$ of the total section area and errors in location of the change in curvature or identification will have only small effect on other measurements. The region enclosed by the large rectangle in Figure 2 shows all phases of interest for an imageenhanced surface section. The image is enhanced in the sense that the ice grains, necks and pore space are given different consistent shades of gray. The ice grains are given a black shade, while the pore space and necks are, respectively, white and an intermediate gray shade. Any blemishes in the surface section are also removed. The following parameters can be directly determined from surface-section measurements.

\section{Number of grains per unit length of test line, $N_{\text {gL }}$}

A number of test lines is spaced evenly across the section, and the total number of grains intercepted by these lines is divided by the total length of the test lines to give $N_{\mathrm{gL}}$. In the surface-analysis program, a test line is displaced parallel to itself across the entire area being analyzed in steps of 0.2 scale markings. At each step, the number of intercepted grains is then counted. A grain is counted every time a grain boundary is intercepted twice by the line. This would be done regardless of whether the grain boundary adjoins pore space or neck phase.

\section{Number of bonds per unit area, $N_{\mathrm{bA}}$}

This is simply the number of bonds in the test area divided by the test area. Each bond is automatically counted as the bond diameter is measured. Bonds that are intercepted by the region boundary are counted as $1 / 2$ and are not measured.

\section{The number of grains per unit area, $N_{\mathrm{gA}}$}

This quantity is similar to $N_{\mathrm{bA}}$. In this case, the number of grains must be counted manually. The grains are actually counted in five separate categories, grains having $0,1,2,3$ or more than three bonds appearing in the surface section.

Air bond and ice-area fractions, $A_{\mathrm{aA}}, A_{\mathrm{bA}}, A_{\mathrm{gA}}$ Area-fraction measurements are simply the total area of the phase of interest divided by the total analysis area. The neck regions are delineated when the bond is measured and appear gray on the section. These measurements are an automatic feature of the analysis program.

\section{Mean two-dimensional bond diameter and harmonic mean of two-dimensional bond diameters, $D_{2}$ and $E$}

The two-dimensional bond diameter $D_{2}$ is the average of all bonds within the analysis region. Those intercepted by the boundary are excluded from this measurement.

The harmonic mean $E$, as given by Fullman (1953), ie

$$
E=\frac{1}{N} \sum_{i=1}^{N} \frac{1}{D_{2 i}}
$$

where $N$ is the number of two-dimensional bonds in the section plane and $D_{2 i}$ is length of the $i$ th bond as it is measured in the surface section. Though $E$ has no readily apparent physical meaning, it is important as a variable in equations for polydispersed spheres and disks. In the work presented, bonds in three dimensions are approximated by disks and it is in this context that $E$ is used.

\section{Bond frequency, $f_{0}, f_{1}, f_{2}, f_{3}$}

These represent the relative frequency of grains with 0 , 1,2 and 3 bonds, respectively. This requires the visual counting of the total number of each category and is the same counting measurement used for $N_{\mathrm{gA}}$.

\section{Density ratio, $\alpha$}

The density ratio is the ratio of ice density $\rho_{\mathrm{i}}$ to the snow density $\rho$. The stereologic relationship for $\alpha$ is given by the following (Underwood, 1970):

$$
\alpha=\frac{1}{A_{\mathrm{gA}}+A_{\mathrm{bA}}} \text {. }
$$

Two types of three-dimensional parameters will be discussed now, those that come directly from the twodimensional values and those that normally require some assumption of a particle-shape factor. Those which need only two-dimensional values are based purely on statistical assumptions and are therefore valid for particles of any shape.

Mean bond radius, $R_{3}$

As given by Kry (1975a), a grain bond is defined to be 
the plane surface of minimum area located in the neck region of two joined grains. The neck regions within the three-dimensional snow structure represent locations of high stress concentration when the bulk material is subjected to external loading. These regions may have large constrictions relative to the grain structure. Since the grain bond is the location of the largest constriction, it will then have the highest stress concentration, thereby making $R_{3}$ and the mean three-dimensional coordination number, $N_{3}$, important parameters for determining the load-bearing ability of the snow. Kry (1975a) suggested that the bonds in three dimensions may be represented by circular disks. Based upon this assumption, the necessary relationship for the bond radius as derived by Fullman (1953) is

$$
R_{3}=\frac{\Pi}{4 E} .
$$

\section{Mean neck length, $h$}

Hansen and Brown (1986) gave the following relation for $h$ :

$$
h=\frac{A_{b A}}{N_{\mathrm{bV}} \Pi r^{2}} .
$$

The term $N_{\mathrm{bV}}$ is the mean number of bonds per unit volume.

\section{Mean pore length, $\lambda$}

The mean pore length is important in characterizing grain mobility of ice grains within the material matrix. Grain mobility is critical in controling the amount of deformation the material can undergo for a given load condition. Pressure-sintering becomes more dominant as $\lambda$ decreases under external loading. $\lambda$ is affected by both pressure-sintering effects and intergranular slip or glide which also helps to decrease pore size. Therefore, pore-size changes and neck-length changes can be used to find a measure of the extent of intergranular glide. The equation given by Fullman (1953) is valid regardless of grain shape, size or distribution and is given as

$$
\lambda=\frac{\alpha-1}{\alpha N_{\mathrm{aL}}} .
$$

\section{Mean number of bonds per unit volume, $N_{\mathrm{bV}}$}

When a load is placed upon snow, not only bond area but also the number of bonds per grain affect the stress concentration at a bond. An increase in the number of bonds prior to an increase in load is similar to increasing the area of a fewer number of bonds. Based on the assumption of bonds being circular disks, Fullman (1953) has derived the necessary relation for determining the mean number of bonds per volume,

$$
N_{\mathrm{bV}}=\frac{8 E N_{\mathrm{bA}}}{\Pi^{2}} .
$$

\section{Mean intercept length, $L_{3}$}

The equation for the mean intercept length, as derived by Underwood (1970), is valid without assumptions of grain shape, size or distribution and is given by

$$
L_{3}=\frac{A_{\mathrm{g} \Lambda}+A_{\mathrm{bA}}}{N_{\mathrm{gL}}} .
$$

$L_{3}$ provides a measure of the grain length. It is useful along with the mean pore length in characterizing the grain mobility.

Mean grain surface area per volume, $S_{\mathrm{V}}$ The equation derived by Hansen and Brown (1986) is

$$
S_{\mathrm{V}}=4 N_{\mathrm{gL}}-2 \Pi R^{2} N_{3} N_{\mathrm{gV}} .
$$

This represents the mean surface area per volume of detached grains minus the mean surface area of the grain bonds.

The parameters that remain to be calculated are, $N_{\mathrm{gV}}$ (the mean number of grains per unit volume), $N_{3}$ (the mean number of bonds per grain) and $V$ (the mean grain volume). These parameters have normally been calculated by making some assumption about grain shape, but attempting to characterize alpine snow by a grainshape factor may not be a valid generalization due to the wide range of shapes present within a given sample. Gubler (1978b) and Hansen and Brown (1986) provided a method for determining these parameters without any assumption of grain shape, other than for an initial guess.

Gubler's method for evaluating $N_{3}$ was based upon several parameters. The first was the probability $p$ that, if a grain with coordination number $N_{3}=1$ is cut by the section plane, its bond lies in the section plane. Next, the distribution of coordination number about the mean coordination number $N_{3}$ was used. This distribution could be changed by an adjustable free parameter $i$, which has no apparent physical meaning. The procedure was then to adjust $N_{3}$ and $i$ until the theoretical values of the two-dimensional distribution given by

$$
f_{2}(i)=N_{2} \sum_{n=1}^{12}\left(\begin{array}{l}
n \\
1
\end{array}\right) p(i-p)^{n-1} f_{3}(n)
$$

converged to the measured values. $N_{2}$ is a normalizing constant introduced by Hansen and Brown (1986) and is given by

$$
\begin{aligned}
& N_{2} \sum_{i=0}^{6} f_{2}(i)=1 \\
& \left(\frac{k}{i}\right)=\frac{k !}{i !(k-i) !} .
\end{aligned}
$$

$f_{3}(n)$ is the three-dimensional probability-distribution function of a grain having coordination number $n$. As derived by Gubler, $f_{3}(n)$ is

$$
f_{3}(n)=N n^{3 i-1} \exp \left(-\beta n^{2 i}\right)
$$

$N$ is a normalizing constant given by

$$
N=4 i \frac{\beta^{3 / 2}}{(\Pi)^{\frac{1}{2}}} .
$$

$\beta$ is given by

$$
\beta=\left[\frac{2}{(\Pi)^{\frac{1}{2}}} \frac{1}{N_{3}} \Gamma\left(\frac{3}{2}+\frac{1}{2 i}\right)\right]^{2 i},
$$

and $\Gamma$ is the gamma function. 
Gubler have the probability $p$ as

$$
p=\frac{s_{\beta}}{s_{\mathrm{k}}}
$$

where

$$
s_{\beta}=\frac{\Pi N_{\mathrm{gL}} b}{N_{\mathrm{gA}}}
$$

and

$$
s_{k}=\frac{4 N_{\mathrm{gL}}}{N_{\mathrm{gV}}} .
$$

$s_{\beta}$ is the area of a band of width $b$ whose center line is the section plane. $s_{\mathrm{k}}$ is the mean grain-surface area. $b$ is given by

$$
b=2\left[R_{3}^{2}-\left(\frac{D_{2}}{2}\right)^{2}\right]^{\frac{1}{2}} .
$$

Hansen adjusted $p$ and $N_{3}$ by varying $N_{\mathrm{gV}}$ and using the equation

$$
N_{3}=\frac{2 N_{\mathrm{bV}}}{N_{\mathrm{gV}}}
$$

which was neglected by Gubler. It is by using Equation (19), and directly varying $N_{\mathrm{gV}}$ and $i$ to force Equation (9) to converge to the measured values, rather than $N_{3}$ and $i$, that Hansen was able to determine $N_{\mathrm{gV}}$ without resorting to the assumption of a shape factor. One problem with Gubler's approach is that he fixed $N_{\mathrm{gV}}$ by assuming a shape factor $C$ and using

$$
N_{\mathrm{gV}}=C \frac{N_{\mathrm{gA}}^{2}}{N_{\mathrm{gL}}} .
$$

This also fixes the value of $N_{3}$ since $N_{\mathrm{bV}}$ is known, yet he still allowed $N_{3}$ to vary which may lead to uniqueness problems. The reader is referred to the papers by Gubler (1978b) and Hansen and Brown (1986) for a more detailed discussion of the above theory.

A slight digression is now in order, and a new definition of the band width $b / 2$ is introduced. In Equation (18), $b / 2$ is the height of a right-triangle necessary to yield a line of length $D_{2} / 2$ lying in the intersecting plane when $R_{3}$, the hypotenuse, is projected on to the plane. Figure 3 illustrates a grain with a band width located about a surface-section plane that contains a bond. Part (b) of that figure provides more detail regarding $b, R_{3}$ and $D_{2}$. The small bonds intersected by the section plane provided the most weight in determining the magnitude of $R_{3}$, with the largest necks having much less influence. This occurs, because $R_{3}$ is based upon the sum of the reciprocal of the two-dimensional bonds (Equations (1) and (3)). With this in mind, it is then reasonable to assume that when $R_{3}$ is projected on to the section plane, one should find those values which most affect its magnitude. The definition as given does not allow for this. Only those values lying between $D_{2} / 2$ and $R_{3}$ will be found within the resulting band width. If this band is narrow, it may happen that all but a small minority of measured necks will lie within the band. By replacing the term in parentheses in Equation (18) with the minimum value found in the section plane, $b$ will then contain most of the necks found in the section plane, particularly those that most heavily influence its magnitude. The

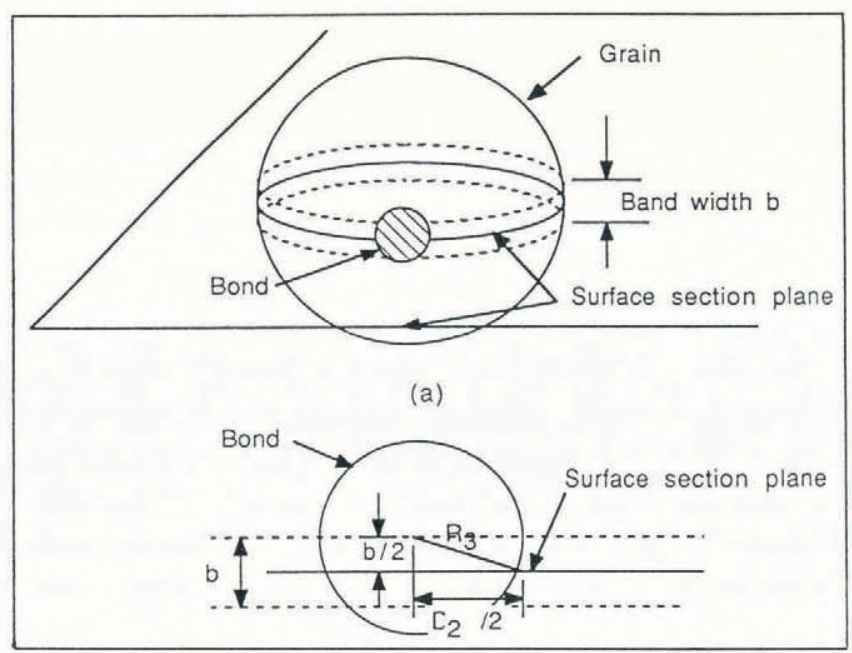

(b)

Fig. 3. Schematic of band width containing a bond exposed on a surface section.

new definition for band width becomes

$$
b=2\left[R_{3}{ }^{2}-\left(\frac{D_{2 \min }}{2}\right)^{2}\right]^{\frac{1}{2}} .
$$

Once $N_{\mathrm{gV}}$ is determined using Hansen's approach, along with the new definition of band width, the mean grain volume can be determined. The governing equation, as given by Hansen and Brown (1986), is

$$
V=\frac{A_{\mathrm{gA}}+A_{\mathrm{bA}}}{N_{\mathrm{gV}}} \text {. }
$$

Hansen included the neck-area fraction, since the neck becomes an integral part of the grain when the bond fractures. This term is important in measuring inertial effects, particularly at high rates of deformation.

\section{EXPERIMENT AND RESULTS}

Freshly fallen snow was collected and stored in a cold room at $-10^{\circ} \mathrm{C}$. Confined compression tests were carried out at $-10^{\circ} \mathrm{C}$ within a period of $2-6$ months after the samples were first placed in the cold room. After 2 months, the original sample volume had decreased by nearly $50 \%$, and volume changes occurring after this were generally very small. The snow samples consisted of snow from 2 years, so that the large-grained samples (sample numbers $10 \mathrm{k} 01,5 \mathrm{k} 001$ and $2 \mathrm{k} 501$ ) were from the earlier year (1986) and the fine-grained samples (sample numbers $5 \mathrm{k} 002$ and $2 \mathrm{k} 502$ ) were from the following year (1987). The initial densities of the samples were all between 250 and $320 \mathrm{~kg} \mathrm{~m}^{-3}$, so there was not a wide spread in this variable among the samples tested. The notation used for identifying samples was:

(1) Maximum load to which a sample would be subjected is indicated by the first four terms $(2 \mathrm{k} 50$, $2500 \mathrm{lb}(11.2 \mathrm{kN}))$.

(2) p or $\mathrm{f}$ indicates if the sample is pre-compressed or in its final compressed state. 
(3) 1 or 2 indicates if the sample was tested in the first or second year, respectively.

The first part of the experiment involved subjecting samples to a constant deformation rate, in this case at the nominal strain rate of $2.75 \times 10^{-3} \mathrm{~s}^{-1}$, until a predetermined maximum load value was reached. The chosen load maxima were $44.8,22.4$ or $11.2 \mathrm{kN}$, corresponding, respectively, to Cauchy stresses of 1.55, 0.77 and $0.387 \mathrm{MPa}$. Once this load was attained, the test was stopped. Surface sections were then immediately prepared from the uncompressed and compressed samples. Surface preparation was based on the procedure given by Perla (1982). Prints of the sections were made with the image as large as possible, while maintaining good contrast and avoiding graininess. The magnifications used ranged between $10 \times$ and $20 \times$. Using the digitizing software supplied with the Thunder Scanner on a Macintosh computer, it was then possible to run the image file through a filter to remove blemishes and maximize contrast. In order for the digitized images to be compatible with the image-analysis software, the section contrast had to be set to make the ice grains black and pore spaces white.

The image scale was first measured. The region to be analyzed was delineated by marking off a rectangular region. Next, each grain bond was measured by marking a rectangular region enclosing the neck. The bond diameter, the plane and neck area were then automatically measured. After all bonds connected to a particular grain had been measured, the number of these bonds was tallied by selecting the value of either $1,2,3,>3$ or $1 / 2$ (for those bonds intersected by the region boundary). After all bonds had been measured, the number of grains not having a bond was tallied and assigned the value of 0 .

Two areas of each image were analyzed. The results were then averaged to obtain mean values and relative errors. The major factors limiting the size of each area analyzed were the actual photograph size and the part

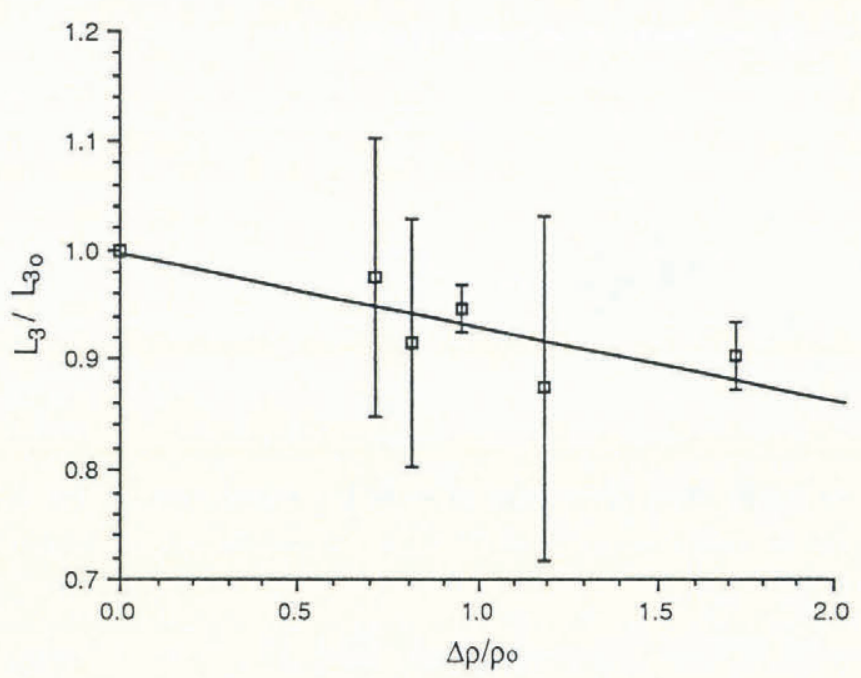

Fig. 4. $L_{3} / L_{3_{0}}$ vs $\Delta \rho / \rho_{0}$. Ratio of mean final and initial mean intercept length versus change in density. The straight line is a linear regression curve with a standard error of $S_{1}=0.032$. The error bands represent the total data scatter.

of the image for which each feature's identity could be accurately determined. The resolution of the digitized images was better than $0.0333 \mathrm{~mm} \mathrm{pixel}{ }^{-1}$.

In the results, it is important to keep in mind that two distinct ranges of grain-size exist. The large grains, corresponding to the number 1 samples, have values of $L_{3}$ ranging from 0.34 to $0.39 \mathrm{~mm}$ in the uncompressed state, whereas the small-grained (number 2) samples range from 0.19 to $0.22 \mathrm{~mm}$ in the uncompressed state. The ratios of $L_{3} / L_{3_{0}}$ vs $\Delta \rho / \rho_{0}$ are plotted in Figure 4. The " 0 " subscript indicates initial value. $\Delta \rho$ is the change in density resulting from the loading. As indicated in Table 1, neck length tends to remain relatively constant throughout the deformation process. An increase in coordination number does occur, however, and

Table 1. Mean intercept length, mean pore length and mean neck length

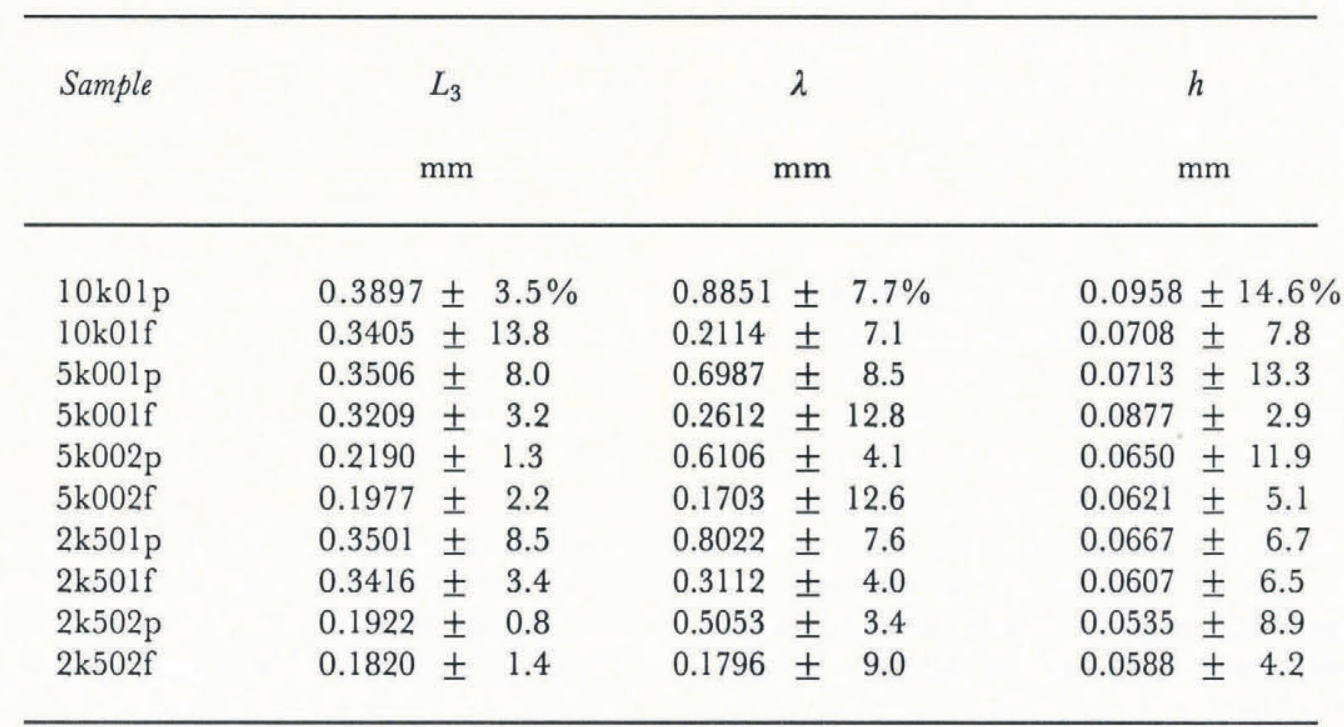


Table 2. Values obtained directly from measured two-dimensional bonds in the plane

\begin{tabular}{|c|c|c|c|c|}
\hline \multirow[t]{2}{*}{ Sample } & $D_{2}$ & $E$ & $R_{3}$ & \multirow{2}{*}{$\begin{array}{c}D_{2 \min } \\
\mathrm{mm}\end{array}$} \\
\hline & $\mathrm{mm}$ & $\mathrm{mm}^{-1}$ & $\mathrm{~mm}$ & \\
\hline $10 \mathrm{k} 01 \mathrm{p}$ & $0.1692 \pm 2.1 \%$ & $7.2841 \pm 3.7 \%$ & $0.1080 \pm 3.7 \%$ & $0.0652 \pm 14.3 \%$ \\
\hline $10 \mathrm{k} 01 \mathrm{f}$ & $0.1299 \pm 0.2$ & $9.2088 \pm 0.1$ & $0.0853 \pm 0.1$ & $0.0434 \pm 4.5$ \\
\hline $5 \mathrm{k} 001 \mathrm{p}$ & $0.1488 \pm 3.9$ & $8.0084 \pm 5.6$ & $0.0984 \pm 5.6$ & $0.0895 \pm 5.0$ \\
\hline $5 \mathrm{k} 001 \mathrm{f}$ & $0.1420 \pm 5.1$ & $9.5567 \pm 6.6$ & $0.0826 \pm 6.6$ & $0.0413 \pm 0.9$ \\
\hline $5 \mathrm{k} 002 \mathrm{p}$ & $0.0899 \pm 5.7$ & $15.6394 \pm 3.4$ & $0.0503 \pm 3.4$ & $0.0178 \pm 21.9$ \\
\hline $5 \mathrm{k} 002 \mathrm{f}$ & $0.0693 \pm 0.4$ & $19.5801 \pm 3.1$ & $0.0402 \pm 3.1$ & $0.0118 \pm 2.5$ \\
\hline $2 \mathrm{k} 501 \mathrm{p}$ & $0.1485 \pm 3.1$ & $7.8755 \pm 0.8$ & $0.0998 \pm 0.9$ & $0.0859 \pm 0.5$ \\
\hline $2 \mathrm{k} 501 \mathrm{f}$ & $0.1315 \pm 9.5$ & $8.5692 \pm 10.1$ & $0.0926 \pm 10.1$ & $0.0703 \pm 9.8$ \\
\hline $2 \mathrm{k} 502 \mathrm{p}$ & $0.0808 \pm 1.9$ & $15.3586 \pm 0.8$ & $0.0512 \pm 0.9$ & $0.0270 \pm 9.1$ \\
\hline $2 \mathrm{k} 502 \mathrm{f}$ & $0.0677 \pm 0.8$ & $18.8240 \pm 2.0$ & $0.0417 \pm 2.0$ & $0.0196 \pm 42.9$ \\
\hline
\end{tabular}

will be discussed later. The factors which collectively govern the change in neck length are fairly complicated, so that predicting neck-length change is difficult. This topic will be discussed in detail in a later paper. It suffices to say that, for the densifications considered here, no definite trends in neck length could be unambiguously determined.

Changes in grain-size did show a definite trend as the material was compressed. Figure 4 illustrates the gradual decrease in grain-size with increasing density. Since the strain rates used in these tests were large, some apparent grain fracture did result. The straight line is the linear regression curve, and the vertical bars represent the entire range of the data scatter.

Table 1 also includes values of $\lambda$, the mean distance across a pore from grain surface to grain surface. Looking

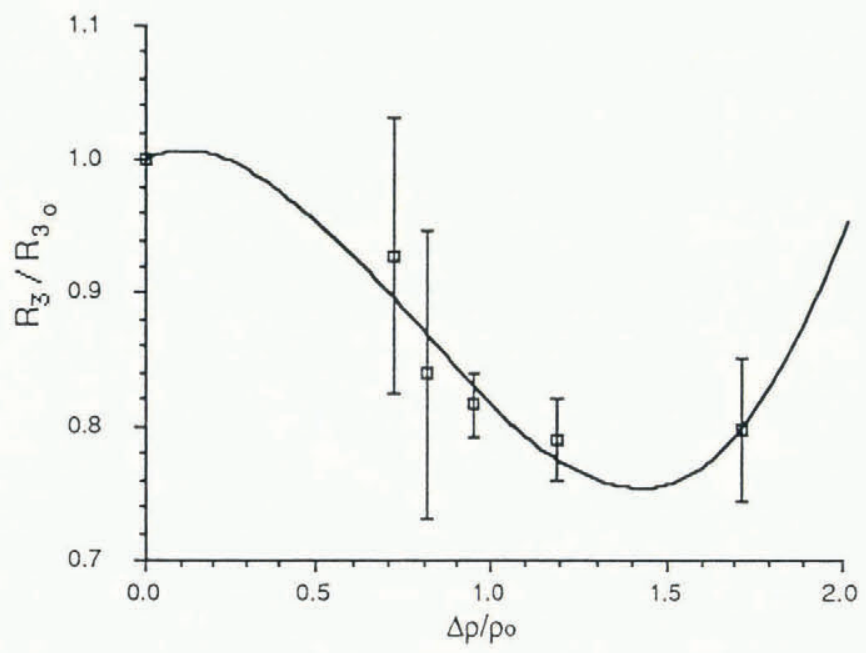

Fig. 5. $R_{3} / R_{3_{0}}$ vs $\Delta \rho / \rho_{0}$. Ratio of final and initial three-dimensional bond radius versus change in density. The curve is a cubic leastsquares fit to the data, and the vertical lines represent the total range of the data scatter. The standard error of the least-squares fit is $S_{R}=0.025$. at the values of $\lambda$ for the compressed samples, the ratio of $\lambda / L_{3}$ decreases from 0.99 to 0.62 as the final stress ranged from 0.387 to $1.55 \mathrm{MPa}$. It is evident that as the grains become more immobile as the load-bearing ability increases.

Two- and three-dimensional bond diameters and radii are given in Table 2. The two-dimensional diameters show a definite decrease from the pre-compressed to compressed state. This indicates that bond growth is less significant than bond fracture and formation of new bonds. In fact, few of these bonds appear to survive the deformation process.

As the grain mobility is decreased, there should be some point at which the rate of bond breakage decreases and an increase in average bond radius begins. Figure 5 shows some indication for this for the largest density change. A complementary factor to the size of the grain bond is the number of bonds per volume $N_{\mathrm{gV}}$. Over the range of loading conditions in this study, the increase in $N_{\mathrm{bV}}$ showed a 2.5 -fold increase at the $11.2 \mathrm{kN}$ load

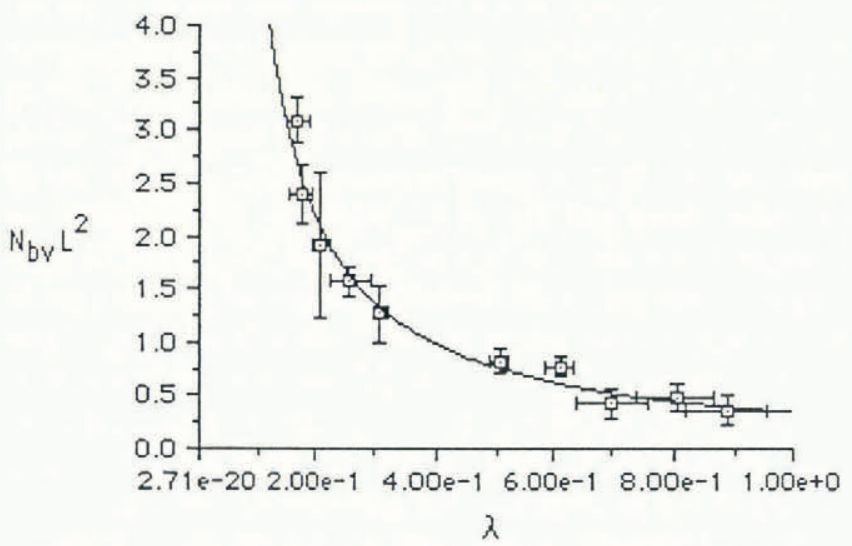

Fig. 6. $N_{b V} L^{2}$ vs $\lambda$. Product of number of bonds per volume and the square of the mean intercept length versus the mean pore length. The standard error for the fit is $S_{N}=0.2$. As in the other figures, the error bands represent the total data scatter. 
Table 3. $N_{3}, N_{\mathrm{gV}}, V$ and $S_{\mathrm{V}}$ for the pre-compressed samples

\begin{tabular}{lccll}
\hline Sample & \multicolumn{1}{c}{$N_{3}$} & \multicolumn{1}{c}{$\mathcal{N}_{\mathrm{gV}}$} & $V$ & \multicolumn{1}{l}{$S_{\mathrm{V}}$} \\
& bonds/grain & \multicolumn{1}{c}{$\mathrm{mm}^{-3}$} & $\mathrm{~mm}^{3}$ & $\mathrm{~mm}^{-1}$ \\
& & & & \\
\hline & & & \\
$10 \mathrm{k} 01 \mathrm{p}$ & $2.28 \pm 10.7 \%$ & $2.09 \pm 4.8 \%$ & $0.15 \pm 4.6 \%$ & $2.80 \pm 17.5 \%$ \\
$5 \mathrm{k} 001 \mathrm{p}$ & $2.41 \pm 7.1$ & $2.89 \pm 5.6$ & $0.12 \pm 5.4$ & $3.41 \pm 6.8$ \\
$5 \mathrm{k} 002 \mathrm{p}$ & $2.64 \pm 3.4$ & $12.19 \pm 3.9$ & $0.02 \pm 6.1$ & $4.31 \pm 8.5$ \\
$2 \mathrm{k} 501 \mathrm{p}$ & $2.58 \pm 10.6$ & $3.05 \pm 2.9$ & $0.10 \pm 2.8$ & $3.00 \pm 13.2$ \\
$2 \mathrm{k} 502 \mathrm{p}$ & $2.36 \pm 9.3$ & $19.22 \pm 6.5$ & $0.01 \pm 6.1$ & $5.01 \pm 2.6$ \\
\hline
\end{tabular}

$(0.387 \mathrm{MPa})$ and 6.9 -fold increase at the $44.8 \mathrm{kN}$ load (1.55 MPa). The values of $N_{\mathrm{bv}}$ are shown in Table 4 .

If the effects of grain-size are factored out, a better correlation between the mean pore length and the number of bonds per volume should exist. To check this, $N_{\text {bv }} L_{3}{ }^{2}$ was plotted versus $\lambda . L_{3}{ }^{2}$ was used to normalize the effects of grain-size. The results are shown in Figure 6. A least-squares curve was fitted to the data. The resulting equation is

$$
N_{\mathrm{bV}} L_{3}{ }^{2}=0.347 \lambda^{-1.153} .
$$

This equation can be rewritten in the form

$$
N_{\mathrm{bV}}=0.347\left[\frac{1}{L_{3}}\right]^{3}\left[\frac{L_{3}}{\lambda}\right]\left[\frac{1}{\lambda}\right]^{0.153} .
$$

Finally, introducing three constants $C_{1}, C_{2}$ and $C_{3}$ in place of 0.347 , the physical meaning of the equation becomes clearer. The resulting equation is

$$
N_{\mathrm{bV}}=C_{1}\left[\frac{1}{L_{3}}\right]^{3} C_{2}\left[\frac{L_{3}}{\lambda}\right] C_{3}\left[\frac{1}{\lambda}\right]^{0.153} \text {. }
$$

It can be seen that, by including the constant with the inverse of the cube of the mean intercept length, the inverse of the average grain volume will result. The inclusion of grain volume certainly makes sense as a factor influencing the number of bonds per volume, since the average grain volume determines the number of grains that can fit into a given volume. The second group of terms measures the grain mobility, which would be strongly influenced by the ratio $L_{3} / \lambda$. As the mean pore length decreases relative to the grain-size $L_{3}$, the probability of grains coming into contact and forming bonds should increase. The last set of terms is a weak function of the pore size, and further experimental data might result with this part being nearly constant. At this point, a conjecture as to its significance will not be made.

The discussion of the terms appearing in Equation (24) indicates the validity of this equation on physical grounds. As a result, Equation (24) should be significant in determining the mean coordination number of a sample undergoing large deformation.

The values of $N_{3}, N_{\mathrm{gV}}, V$ and $S_{\mathrm{V}}$ obtained for each of the five pre-compressed samples are shown in Table
3. The resulting mean coordination number $N_{3}$ for these samples ranged from 2.28 to 2.67 bonds/grain. These values appear to be independent of the mean grain-size. In obtaining values of $N_{\mathrm{gV}}$ for the compressed samples, the following equation was used to obtain a first estimate:

$$
N_{\mathrm{gVf}}=\frac{\rho_{\mathrm{f}}}{\rho_{\mathrm{i}}} N_{\mathrm{gVi}}
$$

where the subscripts f and i refer to initial and final respectively, and $\rho$ is the density.

Figure 7 demonstrates the variation of the threedimensional coordination number, $N_{3}$, for the density ranges covered in the testing program reported here.

Values of $S_{\mathrm{V}}$ in Tables 3 and 4 indicate an increase in the surface area per unit volume. This is shown in Figure 8. As would be expected, $S_{\mathrm{V}}$ increases steadily for much of the deformation where bond breakage is frequent and where there is some grain fracturing. It then levels out as the density approaches $600 \mathrm{~kg} \mathrm{~m}^{-3}(\Delta \rho / \rho$ approaches $1.75-2.0)$. At densities which are higher than

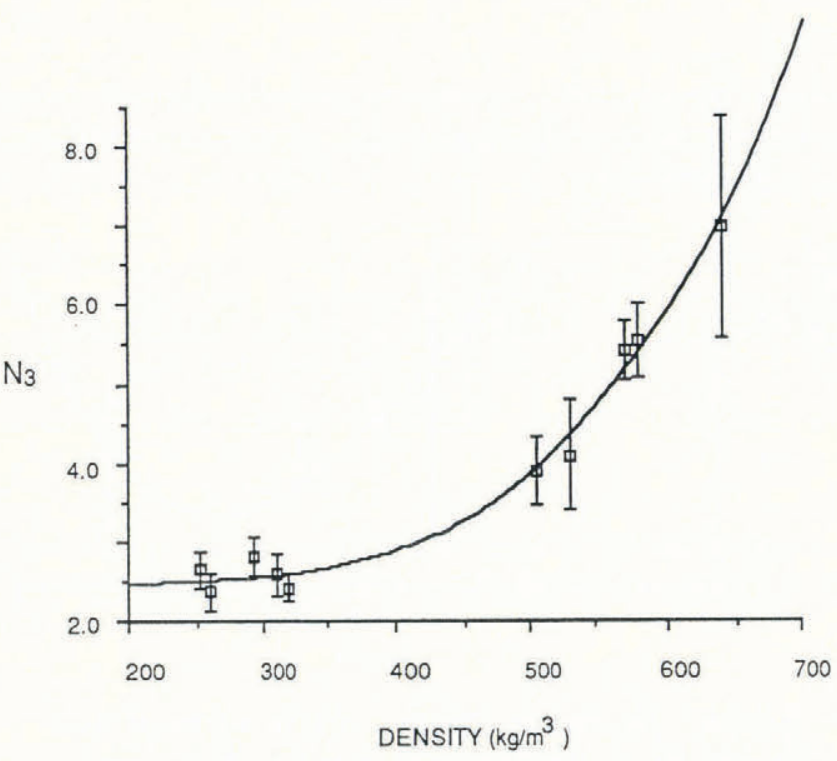

Fig. 7. Three-dimensional coordination number $N_{3}$ vs density. The curve is a least-squares fit to the data with a standard error $S_{N}=$ 0.12. The vertical lines give the total data scatter. 
Table 4. $N_{3}, N_{\mathrm{gV}}, V$ and $S_{\mathrm{V}}$ for the compressed samples

\begin{tabular}{|c|c|c|c|c|}
\hline Sample & $\begin{array}{c}\quad N_{3} \\
\text { bonds/grain }\end{array}$ & $\begin{array}{l}\mathcal{N}_{\mathrm{gV}} \\
\mathrm{mm}^{-3}\end{array}$ & $\begin{array}{c}V \\
\mathrm{~mm}^{3}\end{array}$ & $\begin{array}{l}S_{\mathrm{V}} \\
\mathrm{mm}^{-1}\end{array}$ \\
\hline $10 \mathrm{k} 01 \mathrm{f}$ & $7.14 \pm 19.6 \%$ & $4.74 \pm 13.6 \%$ & $0.14 \pm 4.6 \%$ & $5.83 \pm 13.6 \%$ \\
\hline $5 \mathrm{k} 001 \mathrm{f}$ & $5.85 \pm 8.0$ & $5.24 \pm 6.0$ & $0.11 \pm 5.4$ & $5.60 \pm 7.2$ \\
\hline $5 \mathrm{k} 002 \mathrm{f}$ & $5.72 \pm 6.6$ & $27.69 \pm 4.1$ & $0.02 \pm 3.7$ & $9.32 \pm 7.1$ \\
\hline $2 \mathrm{k} 501 \mathrm{ff}$ & $4.17 \pm 16.8$ & $5.22 \pm 3.0$ & $0.10 \pm 2.8$ & $4.99 \pm 6.1$ \\
\hline $2 \mathrm{k} 502 \mathrm{f}$ & $3.89 \pm 11.9$ & $37.48 \pm 7.0$ & $0.10 \pm 6.1$ & $9.49 \pm 10.2$ \\
\hline
\end{tabular}

those studied here, this ratio should begin to decrease as bond breakage all but ceases, and bond mobility approaches zero.

One should not confuse the results shown in Figure 8 with what one would expect with thermodynamic consolidation of snow. Here, we are studying the large-scale compaction of snow at large enough rates to fracture grain bonds as well as grains. As a consequence, new

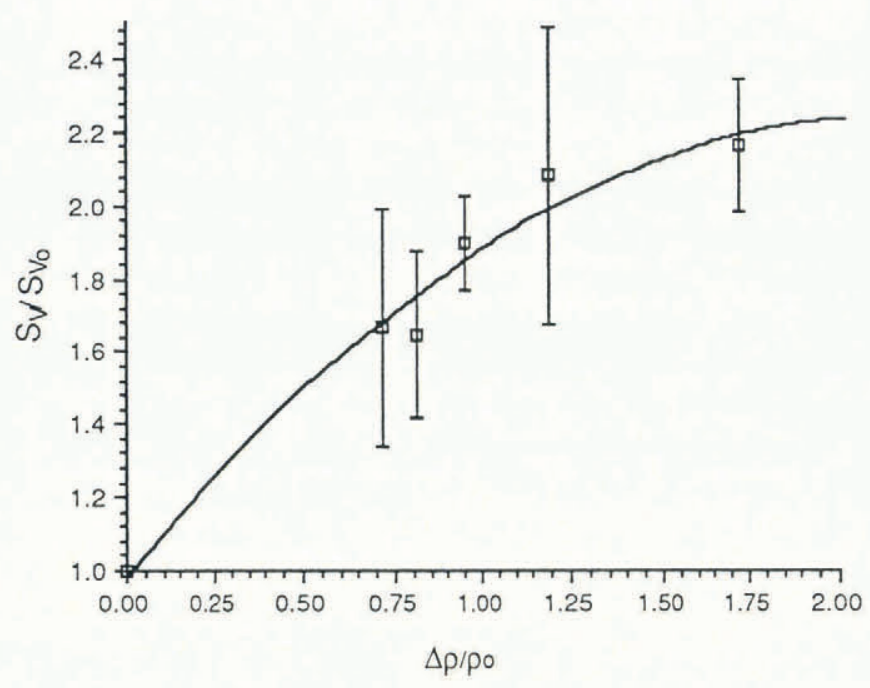

Fig. 8. Variation of normalized surface area per unit volume with change in density, $\Delta \rho / \rho_{0}$. The total data scatter is given by the vertical error bands.

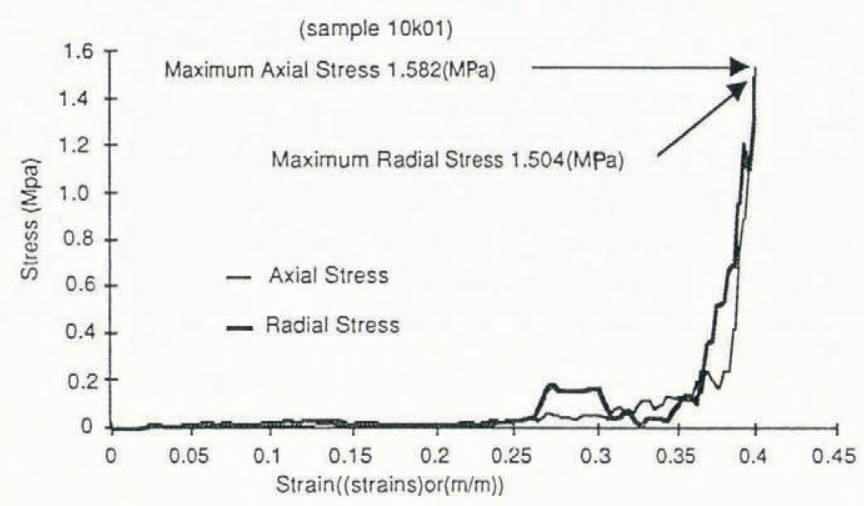

Fig. 9. Axial and radial stress vs Lagrangian strain. surface area is exposed due to the fracturing at high deformation rates. This is in contrast to the slow consolidation of snow as it attempts to reduce its free-surface energy by grain growth and densification. In this case, deformations are so slow that no fracturing occurs, and a gradual reduction of free-surface area results.

\section{DISCUSSION AND CONCLUSIONS}

At the maximum density attained, there is approximately a 3.5 -fold increase in coordination number. The increase in coordination number does not increase very rapidly until densities of $500 \mathrm{~kg} \mathrm{~m}^{-3}$ are reached. This corresponds to strains of approximately $33 \%$. This occurs at the point where the stress response begins increasing dramatically during the compression. The stress is about $0.1 \mathrm{MPa}$ at this point. At the maximum stress, the density $\left(639.4 \mathrm{~kg} \mathrm{~m}^{-3}\right)$ represents a $25 \%$ increase over that at $0.1 \mathrm{MPa}\left(500 \mathrm{~kg} \mathrm{~m}^{-3}\right)$. In this interval, the axial stress increased 16 -fold and the coordination number was doubled. There is approximately a $60 \%$ decrease in the grain mobility as indicated by $\lambda / L_{3}$ at 500 and $639.4 \mathrm{~kg} \mathrm{~m}^{-3}$. The largest relative decrease in bond radius $(22 \%)$ occurs at this maximum stress. This is a $38 \%$ reduction in original bond area, but this is countered by the increased coordination number, with a net increase in bond area by a factor of 1.9 over the entire deformation process. The material stiffness is not a linear function of total bond area, since the structural rigidity of the ice matrix is strongly influenced by the coordination number in the sense that a structurally indeterminant structure is much stiffer than one that is structurally determinant. Therefore, the coordination number is a dominant factor determining the material stiffness and strength. Small changes in microstructural variables such as coordination number can have a measurable effect on the loadbearing ability of snow. As indicated by Brown (1979), the effects of reduced grain mobility due to pore collapse may account for much of this at high densities.

The neck-length parameter $(h)$ has not been described in a satisfactory manner in this study. The results of the testing program and image analysis gave an erratic behavior, as illustrated in Table 1. In some cases, the neck length is seen in increase in value, while in other instances the results showed the neck length decreasing. It was expected that the neck length would decrease with compressive deformation, since compressive stresses on 
the necks would shorten them. Formation of new bonds (and subsequently new necks) during densification would also serve to cause the mean neck length to decrease, since necks with nearly zero length would be added to the averaging process. There are a number of possible explanations for this apparent discrepancy. One could be that an image-analysis system with a better resolution is needed to provide a more accurate measurement of the neck length. Also, the definition of the neck length is difficult to implement and leads to a subjective criterion for measuring $h$. Changes in the sign of the surface curvature as seen in the surface sections is difficult to determine with the system used. This problem is now being studied and will be reported on in a later paper.

There are several experimental results that would complement this study. Values of $L_{3}$ for density changes of $25,50,150$ and $200 \%$ will help verify any trends in grain-size change that are occurring (Fig. 4). It appears that the average grain-bond radius may begin to increase at a density change of $150 \%$ (Fig. 5). Measurements of $R_{3}$ at 130,150 and $200 \%$ are needed to confirm this. The other measurement needed is for the coordination number at a final density of $450 \mathrm{~kg} \mathrm{~m}^{-3}$. This should be sufficient to define the point of transition from low to high coordination numbers. In addition, the methods presented here can also be applied to evaluation of changes in microstructure of snow due to thermodynamic sintering and also for low strain rates where grain and bond fracturing is much less significant.

\section{ACKNOWLEDGEMENTS}

The work reported here was supported by the U.S. Army Research Office (grant No. DAAG29-85-K-0259). The authors wish to express their appreciation of the support by the Geosciences Program in the U.S. Army Research Office.

\section{REFERENCES}

Abele, G. and A. J. Gow. 1975. Compressibility characteristics of undisturbed snow. CRREL Res. Rep. 336 .

Abele, G. and A. J. Gow. 1976. Compressibility characteristics of compacted snow. CRREL Rep. 76-21.

Alley, R. B. 1987. Firn densification by grain-boundary sliding: a first model. J. Phys. (Paris), 48, Colloq. C1, 249-254. (Supplément au 3.)

Brown, R. L. 1979. A volumetric constitutive law for snow subjected to large strains and strain rates. CRREL Rep. 79-20.

Brown, R. L. 1980. A volumetric constitutive law for snow based on a neck growth model. J. Appl. Phys., 51(1), 161-165.

Ebinuma, T. and N. Maeno. 1985. Experimental studies on densification and pressure-sintering of ice. Ann. Glaciol., 6, 83-86.

Fullman, R. L. 1953. Measurement of particle sizes in opaque bodies. AIME, 197, 447-452.

Gubler, H. 1978a. An alternate statistical interpretation of the strength of snow. J. Glaciol., 20(83), 343-357.

Gubler, H. 1978b. Determination of the mean number of bonds per snow grain and of the dependence of tensile strength of snow on stereological parameters. J. Glaciol., 20(83), 329-341.

Hansen, A. C. 1985. A constitutive theory for high rate multiaxial deformation of snow. (Ph.D. dissertation, Montana State University.)

Hansen, A. C. and R. L. Brown. 1986. The granular structure of snow: an internal-state variable approach. J. Glaciol., 32(112), 434-438.

Kry, P.R. 1975a. Quantitative stereological analysis of grain bonds in snow. J. Glaciol., 14(72), 467-477.

Kry, P.R. 1975b. The relationship between the viscoelastic properties and structural properties of finegrained snow. J. Glaciol., 14(72), 479-500.

Maeno, N. and T. Ebinuma. 1983. Pressure sintering of ice and its implication to the densification of snow at polar glaciers and ice sheets. J. Phys. Chem., 87(21), 4103-4110.

Perla, R. 1982. Preparation of section planes in snow specimens. J. Glaciol., 28(98), 199-204.

Underwood, E. E. 1970. Quantitative stereology. Reading, MA, Addison-Wesley.

Wilkinson, D. S. 1988. A pressure-sintering model for the densification of polar firn and glacier ice. J. Glaciol., 34(116), 40-45.

The accuracy of the references in the text and in this list is the responsibility of the authors, to whom queries should be addressed. 